\title{
Initial Results From a CdTe High-Energy X-ray Detector on a TEM
}

\author{
Hendrik O. Colijn ${ }^{1}$, David W. McComb ${ }^{1}$
}

${ }^{1}$ Center for Electron Microscopy and Analysis, The Ohio State University, Columbus, OH, USA

Energy Dispersive X-ray analysis (EDX) in the transmission electron microscope (TEM) is a well-established technique for thin films. The higher accelerating voltage and small analysis volume allow experiments not be possible in the SEM. However, there are instances where characteristic X-rays from different elements are not resolvable using conventional Si based detectors. Detection of higher energy $\mathrm{X}$-rays from these elements may circumvent the overlap issues. CdTe and CdZnTe detectors have been used for a number of years in nuclear and gamma-ray spectroscopy [1]. The energy response of a typical $1 \mathrm{~mm}$ thick detector is shown in Figure 1 [2]. These experiments were intended to see if the ability of a CdTe X-ray detector to see higher energy photons would provide the ability to see and resolve high energy $\mathrm{X}$-ray peaks in the TEM.

Our measurements were performed on a Tecnai F20 operating at 200kV. The detector was equipped with a $4 \mathrm{mil}(100 \mu \mathrm{m}) \mathrm{Be}$ window which limits the low energy response. In future studies, a thinner window will improve the low energy sensitivity of the system.

The detector is able to detect the Uranium $\mathrm{K}$ lines in a Uranyl Acetate stained bacterial sample (Figure 2.) despite having an overvoltage of less than 2 . The overvoltage, particularly for $200 \mathrm{kV}$ microscopes, is something that will have to be taken into consideration when doing analyses. Also for lines near $100 \mathrm{keV}$, the detector efficiency will need to be considered. For the Uranium $\mathrm{K}$ lines, the detector efficiency is about 50\%. Spectra from a high-entropy alloy containing Ti, Zr, Nb, Mo, and Ta (Figures 3a and 3b) clearly resolve all the K-lines from Ti to Ta. The K lines for $\mathrm{Zr}(15.77 \mathrm{keV}), \mathrm{Nb}(16.62 \mathrm{keV})$, and Mo $(17.48 \mathrm{keV})$ are all clearly seen. The observed energy resolution for the TaK $\alpha$ lines is about $700 \mathrm{eV}$.

As the energy of the lines increases, the separation of the $\mathrm{K} \alpha 1$ and $\mathrm{K} \alpha 2$ lines becomes visible as shown in Figure 4. The Eu K $\alpha$ and $\mathrm{K} \beta$ lines are clearly visible at 41.5 and $47 \mathrm{keV}$. Although they are not fully resolved the $\mathrm{Ir} \mathrm{K} \alpha 1$ at $84.89 \mathrm{keV}$ is clearly distinguishable from the $\mathrm{K} \alpha 2$ at $63.29 \mathrm{keV}$.

The detector system is able to distinguish between a W-rich matrix and a Ta-rich ppt in a high-entropy alloy (Figure 5). These are adjacent elements in the periodic table and experience some peak overlap yet the spectra are sufficiently clear that the phases are recognizable.

The CdTe detector shows promise in its ability to use high energy X-ray lines. Further optimization of the detector and electronics should improve the performance of the system. For 200kV TEMs overvoltage issues may limit the ability to measure lines over approximately $70 \mathrm{keV}$.

\section{References:}

[1] RB James, P Siffert, eds., $11^{\text {th }}$ International Workshop on Room Temperature Semiconductors and Associated Electronics, Nucl. Instrum. Methods Phys. Res. A 448 (2001) p.1-603.

[2] http://amptek.com/product/xr-100t-cdte-X-ray-and-gamma-ray-detector/\#5

[3] The authors acknowledge support from The Ohio State University and the Ohio Third Frontier Research Scholar program. 


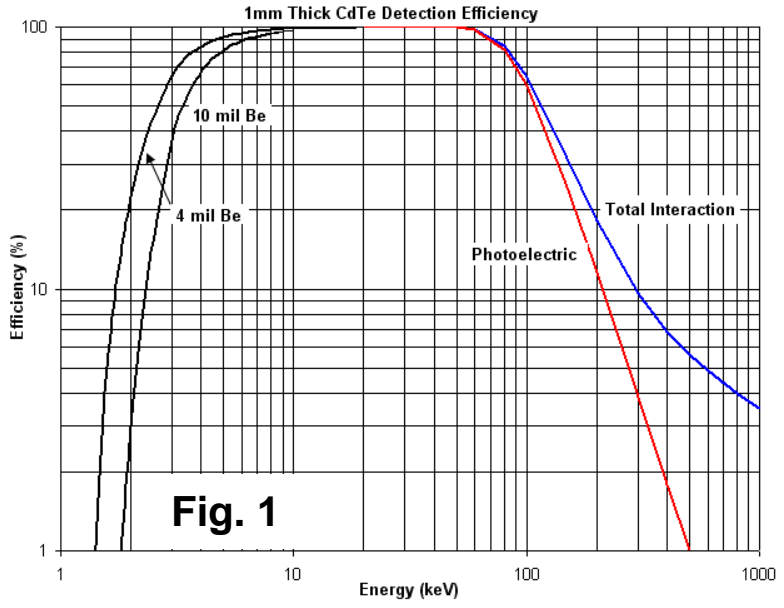

High Entropy Alloy Spectrum

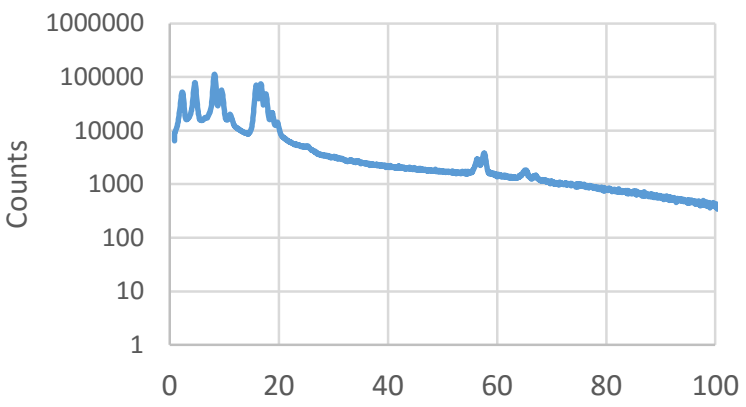

Fig. 3a

Energy (keV)

$\mathrm{EuIrO}_{7}$

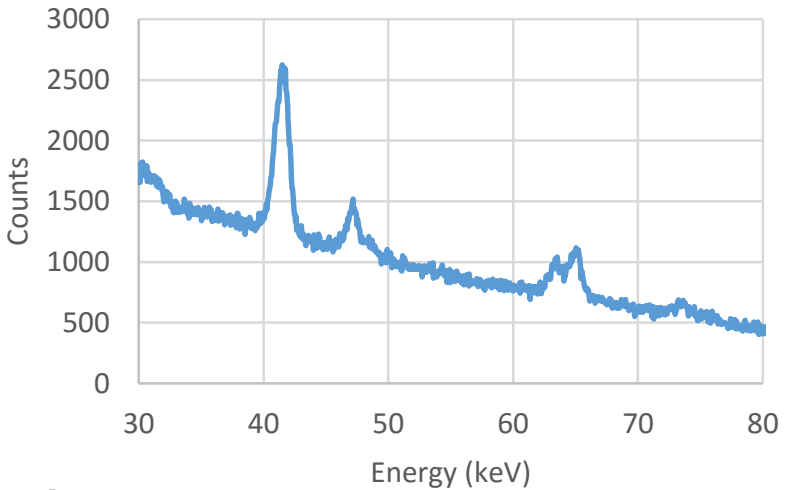

Fig. 4

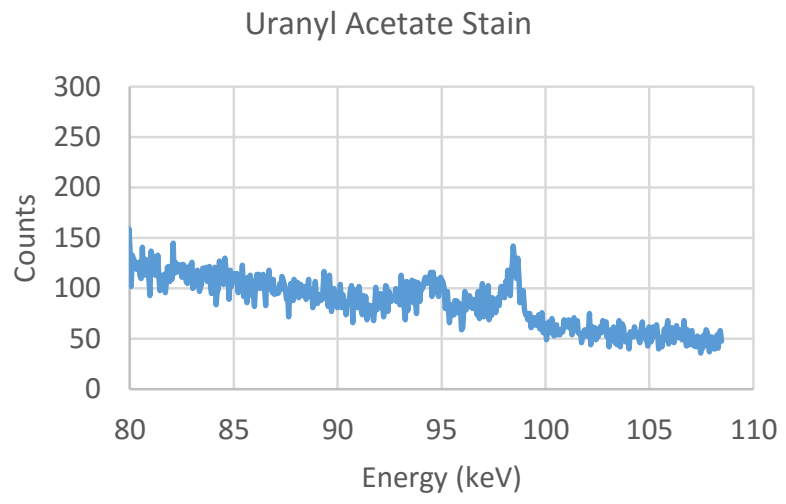

Fig. 2

Ta K series

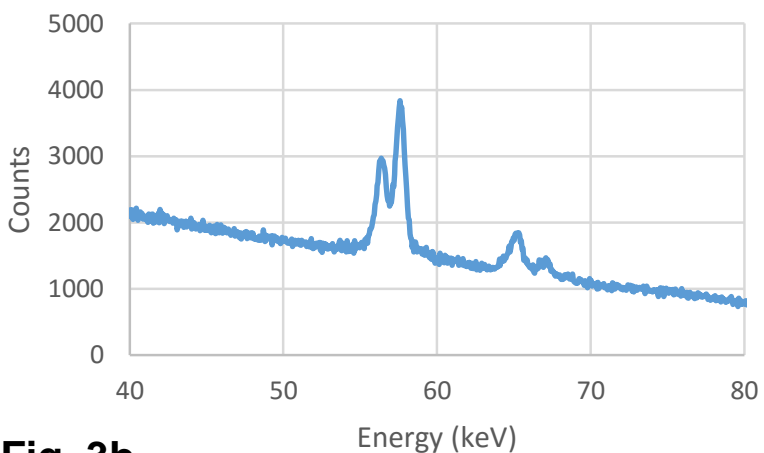

Fig. $3 b$

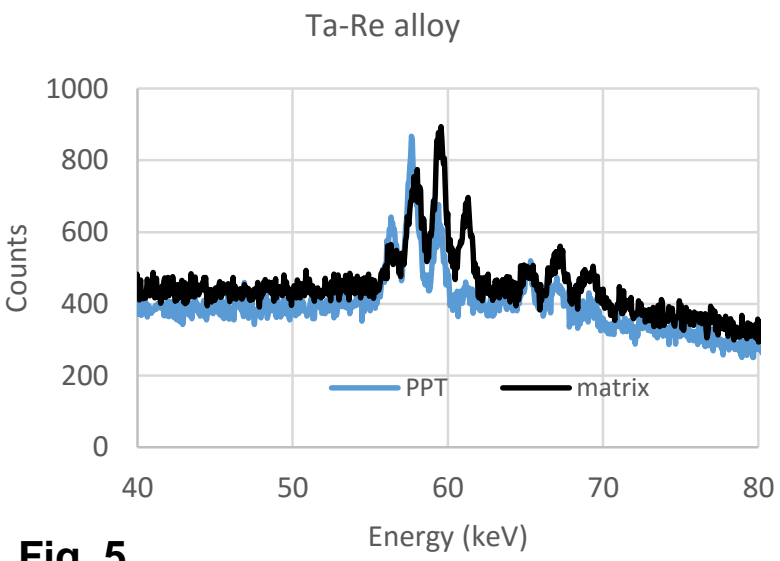

Figure 1. Energy Response of a CdTe detector.

Figure 2. Uranium K lines from a Uranyl Acetate stained sample.

Figure 3. (a) High-Entropy alloy spectrum, (b) Ta peaks from alloy spectrum.

Figure 4. EuIrO7 spectrum showing Eu and Ir peaks.

Figure 5. Comparison Spectrum of phases in a Ta-Re High-Entropy Alloy. 